\title{
Correspondence between the latitudinal ULF wave power distribution and auroral oval in conjugate ionospheres
}

\author{
Martines-Bedenko, V.A. ${ }^{1}$, V.A. Pilipenko ${ }^{2}$, M.D. Hartinger ${ }^{3}$, M.J. Engebretson ${ }^{4}$, \\ D.A. Lorentzen ${ }^{5}$, A.N. Willer ${ }^{6}$ \\ ${ }^{1}$ Institute of Physics of the Earth, Moscow \\ ${ }^{2}$ Space Research Institute, Moscow \\ ${ }^{3}$ Virginia Tech, Blackburn, VA \\ ${ }^{4}$ Augsburg University, Minneapolis, MN \\ ${ }^{5}$ UNIS, Svalbard \\ ${ }^{6}$ National Space Institute, Technical University of Denmark, Copenhagen \\ E-mail: space.soliton@gmail.com
}

Accepted: 10 January 2018

\begin{abstract}
We examine the Pc5 wave power latitudinal distribution in the morning sector along the conjugate magnetometer arrays in Greenland and Antarctica. These distributions are compared with the location of the auroral oval, reconstructed using the OVATION-prime model. This model makes it possible to reconstruct separately the spatial structures of diffuse and monoenergetic precipitation of auroral electrons. Mapping of the spectral power of narrowband Pc5 waves onto the auroral oval has shown that the wave power in the morning sector is localized inside the auroral oval, namely at the poleward edge of diffuse precipitation, but near the peak of monoenergetic precipitation. The case analysis results have been confirmed by a larger statistical study. This observational result confirms the effects earlier found in case studies: the spatial/temporal variations of Pc5 wave power are closely related to the location of the auroral electrojet and magnetospheric field-aligned currents. From the observed relationship between the wave power and the auroral boundaries, it may be concluded that the poleward edge of the diffuse precipitation, around the maximum of the monoenergetic precipitation, is preferred latitude of magnetospheric field-line resonance excitation. This effect is not taken into account by modern theories of ULF Pc5 waves.
\end{abstract}

(c) 2018 BBSCS RN SWS. All rights reserved

Keywords: ULF waves, auroral oval, OVATION model, electron precipitation

\section{Introduction}

At high latitudes of near-Earth space, there are two well-known electrodynamic phenomena, which so far have been considered independently: the auroral oval and ultra-low-frequency (ULF) waves. The auroral oval is a region of auroral emission caused by precipitation of suprathermal electrons. The oval boundaries are in fairly constant motion; during storm time the equatorward boundary moves to lower latitudes, and it travels poleward during storm recovery phase. The poleward boundary of the nighttime auroral oval is very dynamic and shows permanent irregular intensification, even during geomagnetically quiet times [Lyons et al., 1999]. The elevated ionospheric conductivity in the auroral oval region provides a latitudinally confined channel for the auroral electrojet in the westward direction on the dawnside, and in the eastward direction on the duskside. The auroral electrojet constitutes the ionospheric part of the 3D magnetosphere-ionosphere current system driven by the solar wind-magnetosphere interaction.

Geomagnetic ULF waves in the Pc5 band $(f \sim 2-7$ $\mathrm{mHz}$ ) are easily observed due to their large amplitudes (up to some $100 \mathrm{nT}$ ) and long periods. Pc5 pulsations are the most powerful electromagnetic wave process in geospace; their signatures can be observed in space (with magnetometers, electric field probes, and particle detectors), in the ionosphere (with radars, riometers, and auroral imagers), and on the ground (with magnetometers and telluric sensors). Despite the long history of studies of the Pc5 nature and possible excitation mechanism, the physics of these waves has not yet been finally established. Several types of wave activity are observed in the Pc5 frequency range on the ground and in space, so different mechanisms may contribute to magnetospheric wave generation in the same frequency band. Broadband Pc5 disturbances are observed during the storm preliminary phase [Kleimenova et al., 1997] and main phase [Schott et al., 1998], while quasi-monochromatic Pc5 waves occur during the recovery phase [Posch et al., 2003]; specific dayside broadband Pc5-6 pulsations are a persistent feature of high latitude regions. These pulsations observed on the ground are associated with azimuthally large-scale toroidal Alfven mode, while small-scale poloidal Alfvenic oscillations excited at the storm recovery phase are screened by the ionosphere from ground magnetometers.

The peculiar latitudinal amplitude-phase structure of ground Pc5 waves [Walker et al., 1979; Ziesolleck et al., 1994] agrees well with predictions from the field line resonance theory, suggesting that these waves are produced by the localized Alfven standing waves in the magnetospheric resonator excited by MHD disturbances from remote parts of the magnetosphere. According to this notion, the position of the Pc5 amplitude peak corresponds to the L-shell where the local Alfven frequency, $f_{A}(L)$, matches the frequency of an external disturbance, $f$, i.e. $f \sim f_{\mathrm{A}}$ [Tamao, 1965; Chen and Hasegawa, 1974; Southwood, 1974]. Therefore, the latitude where the Pc5 power maximizes is to be determined only by spectral features of a driver and the radial distribution of the magnetospheric plasma density. The ionospheric conditions can influence only 
Table. Geographic and geomagnetic locations of the Antarctic stations and conjugate points (*) of Greenland stations

\begin{tabular}{|c|c|c|c|c|c|}
\hline \multirow{2}{*}{$\begin{array}{l}\text { Antarctica } \\
\text { Conjugate } \\
\text { Greenland }\end{array}$} & \multicolumn{2}{|c|}{ Geographic } & \multicolumn{2}{|c|}{ Geomagnetic } & \multirow{2}{*}{ MLT } \\
\hline & Lat. & Long. & Lat. & Long & \\
\hline PG1 & -84.50 & 77.20 & -77.26 & 37.33 & 2.35 \\
\hline UMQ* & -83.99 & 61.26 & & & \\
\hline PG2 & -84.42 & 57.95 & -75.53 & 39.05 & 2.22 \\
\hline $\mathrm{GDH}^{*}$ & -84.74 & 48.41 & & & \\
\hline PG3 & -84.81 & 37.63 & -73.43 & 35.95 & 2.36 \\
\hline ATU* $^{*}$ & -84.65 & 33.29 & & & \\
\hline PG4 & -83.34 & 12.25 & -71.08 & 36.13 & 2.39 \\
\hline SKT $^{*}$ & -83.11 & 10.27 & & & \\
\hline
\end{tabular}

based on the DGRF/IGRF for epoch 2015

(https://omniweb.gsfc.nasa.gov/vitmo/cgm_vitmo.html)

the damping factor of the field line magnetospheric resonator.

Thus, according to the existing knowledge, the latitudes of the auroral oval location and the Pc5 power peaks are determined by entirely different processes and should not be directly related to each other. However, Lam and Rostoker [1978] noticed that the positions of the westward auroral electrojet and Pc5 power were observed in the same latitude range and both varied in a coherent way. The close association of Pc5 wave latitudinal maximum and position of the auroral electrojet was confirmed for several cases by Pilipenko et al. [2001]. A multiple regression analysis [Simms et al., 2006] showed that Pc5 spectral power increased at those ground magnetometers under the aurora, as determined from the DMSP particle data. The auroral oval boundaries estimated using the database of UV observations of the aurora by the IMAGE satellite has been mapped onto the Pc5 wave power spatial distribution [Kozyreva et al., 2016]. The "epicenter" of the spectral power of broadband fluctuations in the Pc5 band during storm main phase turned out to be mapped inside the auroral oval, whereas during the recovery phase the spectral power of narrowband Pc5 waves was mapped around its equatorward boundary.

The location of the auroral oval (or its equatorward border) was found to be a preferred latitude of magnetospheric field-line Alfven resonator excitation. However, the events presented in earlier papers were case studies and they may be considered as a mere accidental coincidence of the ULF latitudinal maximum and a wide auroral oval. Therefore, this relationship should be examined in a more depth. The problem of a possible relationship between the Pc5 excitation region and the relevant magnetospheric domain and its significance to generation mechanisms of ULF waves in the magnetosphere has not been investigated further.

In this paper we analyze a possible coupling between the auroral oval and Pc5 wave activity. The auroral particle precipitation spatial structure is reconstructed with the help of the OVATION-prime model, based on the DMSP low-orbit measurements of precipitating electrons. The Pc5 activity in both hemispheres is observed with the latitudinal arrays of

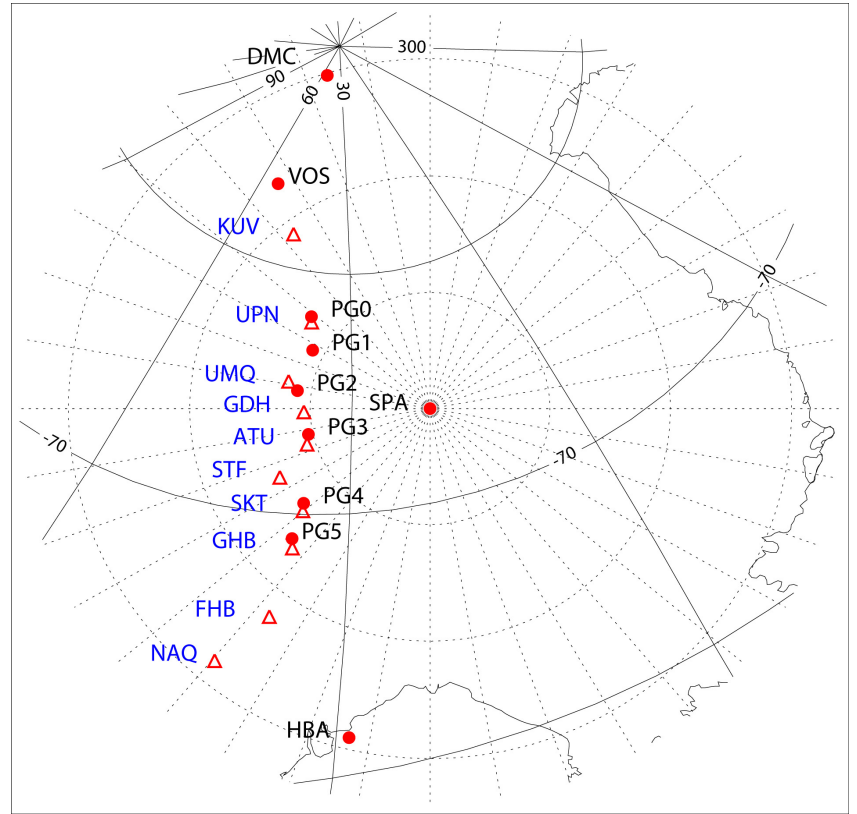

Figure 1. A map of the Antarctic magnetometer stations (red dots) used in this study. The geomagnetic projection of Greenland stations is shown by empty triangles (red). Dotted lines show geographic coordinates, and solid lines denote corrected geomagnetic coordinates.

conjugate magnetometers in Antarctica and Greenland. This enabled us to visualize the spatial location of the auroral oval and Pc5 power, and to examine the dynamics of the auroral oval and ULF activity in greater detail. We finally consider the significance of these observations for current ULF wave physics.

\section{Space weather database and data analysis technique}

\section{Antarctica-Greenland magnetometer arrays}

The Magnetosphere-lonosphere Science Team at Virginia Tech developed an autonomous adaptive low-power instrument platform (AAL-PIP) to establish a ground instrument network with sampling cadence $1 \mathrm{~s}$ in remote field locations in Antarctica, magnetically conjugate to the Greenland East coast magnetometer chain along the $\Lambda \sim 40^{\circ}$ magnetic meridian. Figure 1 shows the locations of the AAL-PIP stations and the geomagnetic conjugate points of their counterparts in Greenland. Conjugate pair coordinates are given in the Table 1.

The Greenland Coastal Array is equipped with three-axes linear-core fluxgate magnetometers which run fully automatically and are optimised for long-term stability. The sensor axes are oriented along local magnetic north (Bx), local magnetic east (By), and vertical down (Bz). 10-sec data were available for the period under study.

The magnetometer data have been high-pass filtered with a cut-off frequency of $1 \mathrm{mHz}$. Spectral analysis is performed in a running 20-min window. The spectral amplitude is estimated with the periodogram method and measured in $\mathrm{dB}$, as $20 \log _{10}(\mathrm{~S})$. 


\section{Auroral Boundaries}

A source of information on the location of the auroral oval is the OVATION-prime (OP) model (sdwww.jhuapl.edu/Aurora/ovation_live//). The OP model is based on energetic particle measurements from the polar-orbiting Defense Meteorological Satellite Program (DMSP) satellites and considers main types of auroras: discrete electron monoenergetic aurora and broadband diffuse aurora [Newell et al., 2009]. Diffuse aurora is primarily caused by the precipitation of energetic electrons $(0.1-30 \mathrm{keV})$ over a broad latitude range. Although generally not visible to the naked eye, the diffuse auroral precipitation provides the main energy source for the high-latitude upper atmosphere (Newell et al., 2009). The discrete aurora at higher latitudes $\sim 77^{\circ}-78^{\circ}$ is dominated by soft electron precipitation $(0.1-1 \mathrm{keV})$. The statistics of wave precipitation (Alfvenic aurora) is small and has a large dispersion, so it was not analyzed here. 11 years of DMSP electrostatic analyzer data have been binned by MLT and geomagnetic latitude and by solar wind and IMF parameters. DMSP data is not affected by sunlight or darkness, and sensitivity of the particle data is many times greater than is possible from either ground-based or space-borne imagers. The equatorward boundary used in OVATION-prime is the equatorward edge of the soft electron precipitation boundary defined by Newell et al. [1996]. The poleward boundary used in OP is intended to be the open/closed boundary.

\section{ULF activity on the ground and in space}

In this study we concentrate on monochromatic Pc5 waves in 2014 in the morning sector. We consider in detail a typical event, when quasi-monochromatic long-lasting Pc5 waves are recorded in both hemispheres.

\section{Jan. 16, 2014 (day 016) event}

Quasi-monochromatic Pc5 waves were excited during 0830-1230 UT at the recovery phase of a weak substorm ( 200 nT) onset 07 UT (Figure 2). The most regular monochromatic waves were observed from 1000 to 1230 UT. In the Northern hemisphere (Greenland) Pc5 pulsations were polarized in the NS direction, that is Bx>>By. At Antarctic stations the contrast between $B x$ and $B y$ is not so pronounced.

Correspondence between the morning auroral oval and Pc5 wave power is examined for the 20-min time interval centered at 1040 UT (Figure 3). The colorcoded station's locations denote Pc5 wave power at each station. The wave activity is characterized by the spectral power (X- and Y-components) bandintegrated in the 2-7 MHz frequency range. The station locations have been superposed onto the auroral particle precipitation pattern derived from the OP model for diffuse (Figure 3, left-hand panel) and monoenergetic (Figure 3, right-hand panel) precipitation. In both hemispheres maxima of Pc5 power (black dots) concentrate near the poleward boundary of the diffuse precipitation, and in the central part of the monoenergetic precipitation.

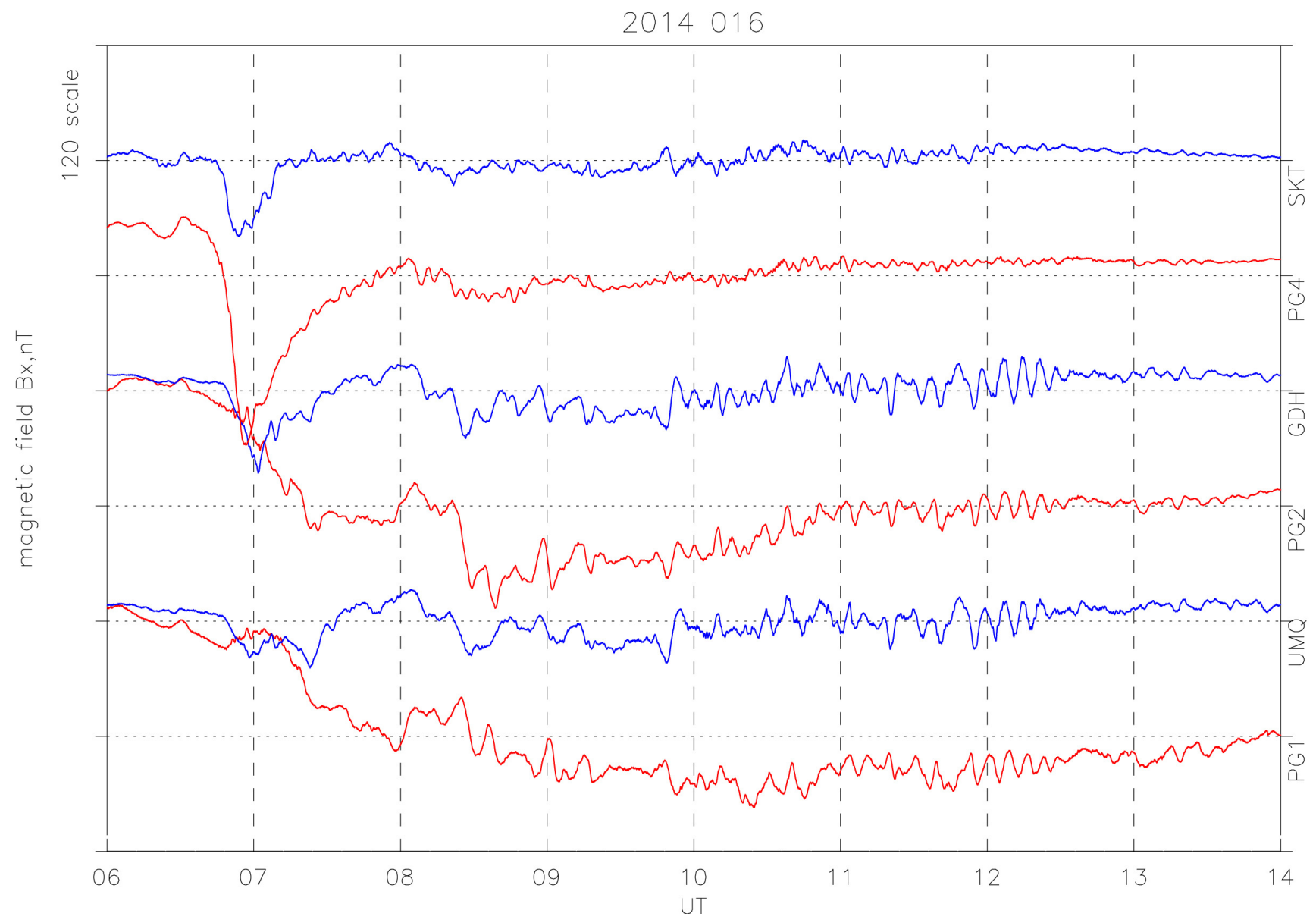

Figure 2. Magnetograms (Bx component) of narrowband Pc5 pulsations during Jan. 16, 06:14 UT along a longitudinal profile in Greenland (blue lines) and Antarctica (red lines). The scale between horizontal ticks is $120 \mathrm{nT}$. 


\section{4/016 1040 UT}

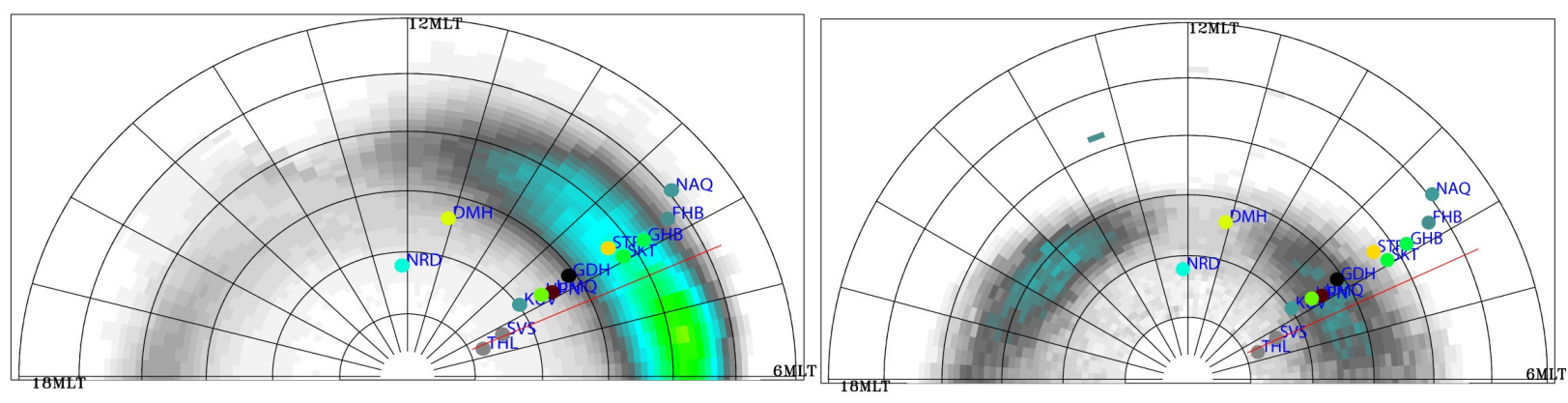

Northen hemisphere

Diffuse

\section{Monoenergetic}

Southern hemisphere
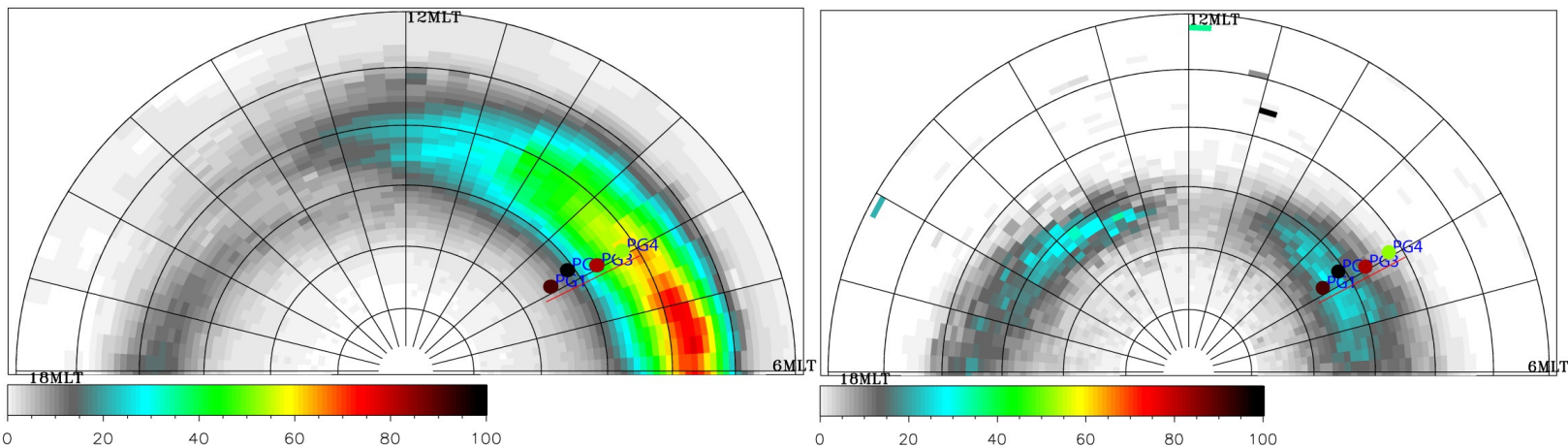

Figure 3. The spatial pattern in geomagnetic coordinates of diffuse auroral precipitation (left-hand panels), and monoenergetic auroral precipitation (right-hand panels), reconstructed with the OP model, during Jan. 16, 2014 at 1040 UT with superposed locations of magnetic stations: Northern hemisphere (upper panels); and Southern hemisphere (bottom panels). Station marks are color coded according to Pc5 spectral power. The color bar below the lower two figures apply to relative intensities (0-100 scale) of both auroral image and ULF wave power (in 20 min window) at each station. The maximal scale of disturbances during analyzed interval are: $B \max =10 \mathrm{~dB}$, and fluxes intensities $\mathrm{Ee}=2.59$ (Northern hemisphere) and 1.47 (Southern hemisphere).

The spectra of the Bx component (not shown) demonstrate the occurrence of a spectral peak at 2 $\mathrm{mHz}$ at most stations in both Northern and Southern hemispheres. We examine the latitudinal structure of the spectral power of monochromatic Pc5 waves at this frequency. The By component is much weaker than the Bx component. The latitudinal distribution of Bx spectral power along the profile has a maximum at geomagnetic latitude $\Phi \sim 75^{\circ}$ in Northern hemisphere (Figure 4, bottom panels). Probably, Pc5 power in Southern hemisphere peaks at the same latitude, but the number of high-latitude Antarctic stations is insufficient to prove it. Signatures of field line resonance can be seen even from visual inspection: localized amplitude of magnetic Bx variations, apparent poleward propagation (not shown), and dominance of the $\mathrm{Bx}$ component over the By component.

In both hemispheres the diffuse precipitation (solid lines in the upper panel) is centered at lower latitude $\sim 71^{0}$ than the monoenergetic precipitation, $\sim 760$ (dotted lines). ULF spectral power is concentrated in the central part of the monoenergetic precipitation (CGM latitude $\sim 75^{\circ}$ ), and around the poleward edge of the diffuse precipitation.

\section{Statistical latitudinal distributions of Pc5 wave power and auroral electron precipitation}

To verify the conclusions made upon the analysis of case studies, we have constructed statistical distributions of the auroral electron flux intensity and Pc5 power. The statistical analysis comprises 20-min time intervals during days with clear long-lasting Pc5 activity: 02/24, 04/04,04/19,06/11, and 12/30 (in total 16 selected time intervals).

All the latitudinal distributions of the auroral electron fluxes during time intervals when Pc5 had been detected are summed up. Each distribution has been normalized to the maximal value and shifted to the reference latitude $74^{\circ}$. The corresponding normalized latitudinal distributions of Pc5 power have been shifted in latitude accordingly. The summary distributions for Northern and Southern polar caps are presented in Figures 5 and 6 .

Diffuse precipitation in both hemispheres has a wide $\sim 10^{0}$ latitudinal peak (Figure 5). The Pc5 wave power distribution is more localized in latitude, around 50. The comparison of these distributions shows that Pc5 power in both hemispheres is located at the poleward edge of the auroral diffuse precipitation, shifted from its maximum by $\sim 50$. 
1.16(016).2014 10:40, longitude: 40.0, 27.5 degrees 1. $-20 . \mathrm{mHz} 2.0$
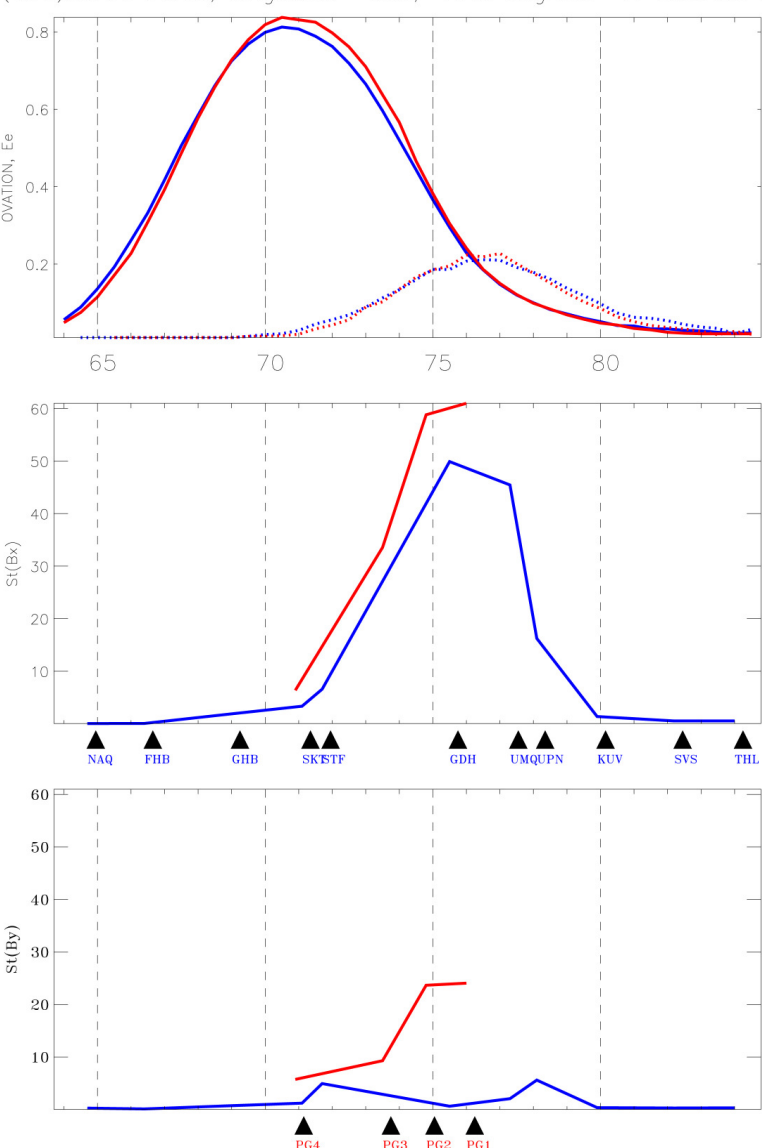

Figure 4. Latitudinal profiles along $\Lambda=40^{\circ}$ of the auroral precipitation electron fluxes: diffuse auroral precipitation (solid lines), and monoenergetic auroral precipitation (dotted lines), derived from the OP model (upper panel), and spectral power density at selected frequencies in the Bx and By components (lower two panels) for the Northern (blue lines) and Southern (red lines) hemispheres during 2014-016, 10:40 $\div 00: 10$ UT. The position of stations in magnetic latitude is marked by filled triangles.

Monoenergetic precipitation has a more narrow latitudinal distribution, with width $\sim 8^{0}$ (Figure 6). The maximum of Pc5 power in both hemispheres is localized in the central region of the monoenergetic electron precipitation.

These results confirm statistically the conclusions made from case studies.

\section{Correspondence between the ULF power and auroral oval:}

\section{Inference for Pc5 wave generation mechanisms}

The occurrence rate and intensity of Pc5 waves has a strong maximum in the morning sector, and a weaker one in the afternoon sector. The azimuthal phase propagation and the polarization features of the ground magnetic disturbance indicate tailward propagation along both flanks of the magnetosphere. These observations led to the conclusion that the Kelvin-Helmholtz instability at morning and dusk flanks is a most likely candidate for the Pc5 generation source. Impulsive or periodic variations of the dynamic pressure of the solar wind [Kessel et al., 2004], and magnetosheath transients [Hartinger et al., 2013] also constitute a possible source of Pc5 wave packets in the magnetosphere. The wave energy is further transported by large-scale wave mode (either cavity, waveguide, or surface modes) from the magnetosphere boundary into inner regions. Upon the wave energy transfer, the wave field experiences a localized frequency-dependent enhancement on a resonant magnetic shell. The existence of resonance effects for ULF geomagnetic pulsations is commonly considered as an indicator of wave energy transfer from an external source towards localized field line Alfven oscillations.

Direct consequences of the field line resonance are the latitudinal mode localization, and the phase shift of $\sim \pi$ of "resonant" magnetic component Bx across the resonant magnetic shell. The amplitude maximum position depends on frequency (a resonant frequency increases towards low $L$ values). The apparent meridional phase velocity in the vicinity of the amplitude maximum is directed poleward in the region where the Alfvenic period grows with latitude. The
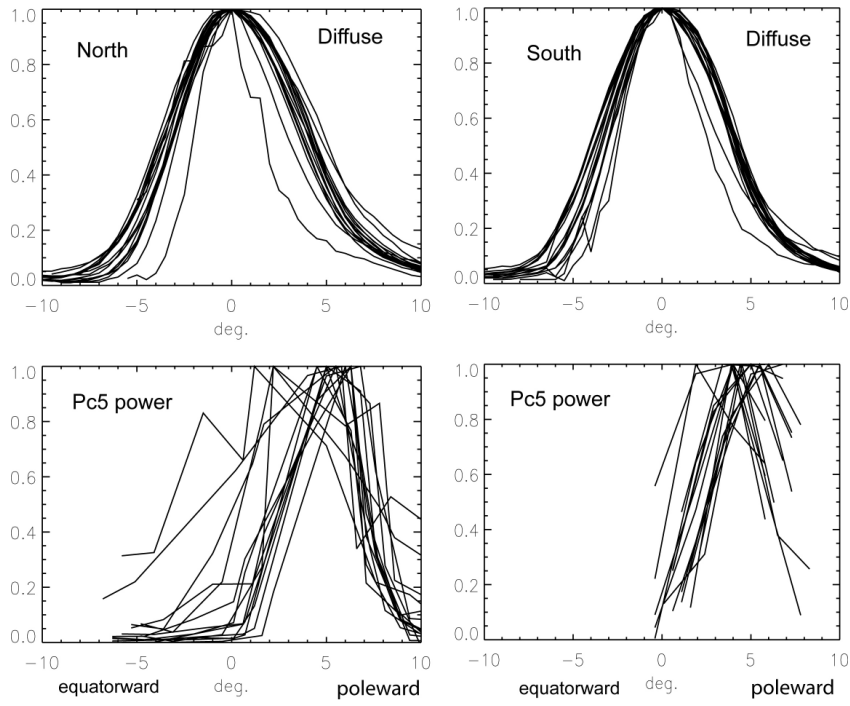

Figure 5. Left-hand panel: Normalized statistical distributions of the diffuse electron precipitation (upper) derived from the OP model and Pc5 wave power at the frequency of the spectral maximum (bottom) in the Northern hemisphere. Right-hand panel: The same, but for the Southern hemisphere.
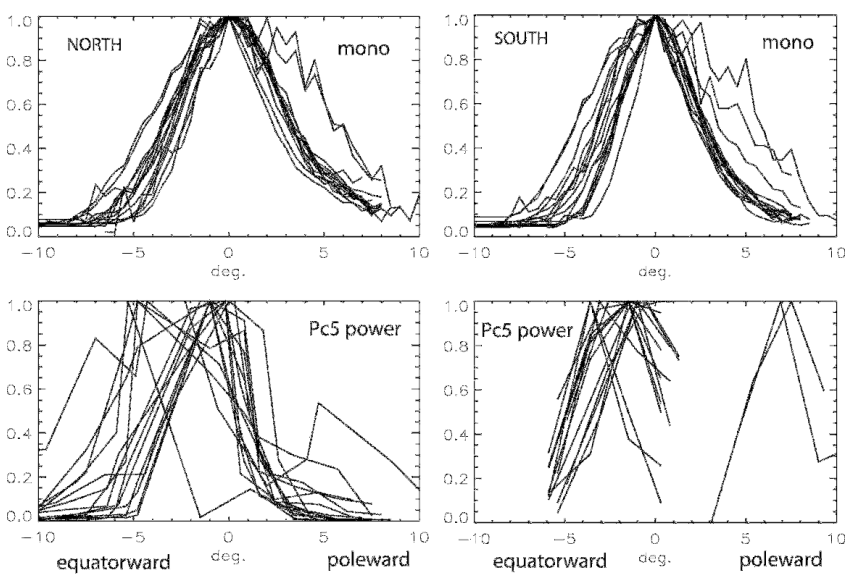

Figure 6. Left-hand panel: Normalized statistical distributions of the monoenergetic electron precipitation derived from the OP model (upper) and Pc5 power at the frequency of the spectral maxima (bottom) in the Northern hemisphere. Righthand panel: The same, but for the Southern hemisphere. 
amplitude and phase spatial distributions of Pc5 clearly demonstrate resonance properties, that have been validated many times with ground magnetometers [Ziesolleck and McDiarmid, 1994], radars [Walker et al., 1979], and optical cameras [Samson et al., 2003].

From these notions on the magnetospheric Pc5 wave generation, it follows that the auroral oval seemingly does not influence wave excitation. Nonetheless, Lam and Rostoker [1978] and later Pilipenko et al. [2001] presented events where the positions of the westward auroral electrojet and the Pc5 wave power were closely linked to each other. Lepidi and Francia [2003], analyzing a statistical latitudinal distribution of low-frequency $(1-4 \mathrm{mHz})$ geomagnetic fluctuations, concluded that the latitude of the maximum power can be considered as an indicator of the auroral oval position. Kozyreva et al. [2016] found that during the storm recovery phase the spectral power of narrowband Pc5 waves was mapped inside the auroral oval. Thus, the location of the auroral oval was found to be a preferred latitude of magnetospheric field-line Alfven resonator excitation. Moreover, it was suggested that the auroral oval associated with a high level of field-aligned current fluctuations, precipitation-modified ionospheric conductivity, strong convection flow, etc. can play an active role in the Alfven wave excitation [Pilipenko et al., 2016].

The events analyzed in this paper confirm that the auroral oval favors excitation of Alfven toroidal oscillations by broadband external disturbances in certain region of the magnetosphere. Using the OP model, we have found that Pc5 latitudinal maximum is far poleward of the diffuse precipitation, and close to the peak of monoenergetic auroral electron precipitation, on its equatorward edge. If Pc5 wave resonance region and the auroral oval would be entirely independent from each other, a latitudinal distribution of Pc5 power would be chaotic in respect to the auroral precipitation structure. Nonetheless, observations demonstrate an occurrence of a certain coordination between the Pc5 latitudinal profile and the auroral electron precipitation domains. Though some theoretical interpretations of the auroral oval Pc5 wave coupling were suggested [e.g., Rostoker and Lam, 1978; Sutcliffe and Rostoker, 1979; Pilipenko et al., 2016], a responsible mechanism has not been reliably revealed. Notice that the electron precipitation patterns related to the wave energization of auroral electrons (Alfvenic aurora) are rather rare and they have not been included in the analysis.

The close association of the auroral oval and resonant Pc5 pulsations is not taken into account by modern theories of ULF waves, but it could be significant for the development of more adequate models. The dynamics of the ULF wave activity "epicenter" during magnetic storms is important not only for the physics of ULF waves, but for understanding of the formation of the outer radiation belt. The transfer of considerable energy of Pc5 waves to a small group of magnetospheric electrons via wave resonant interactions while drifting around Earth was suggested as a possible mechanism of radiation belt acceleration of electrons up to relativistic energies [Mann et al., 2012]. Therefore, the knowledge of the dynamics of
Pc5 wave power latitudinal structure and its relevance to basic magnetospheric domains are critically important for validation of models of radiation belt electron energization.

Here we do not consider the problem of conjugacy and symmetry of Pc5 waves at high latitudes. The evident in-phase relationships between $B x$ components of Pc5 waves in opposite hemispheres (Figure 2) indicates that Pc5 field-aligned structure corresponds to fundamental mode with symmetric electric field in respect to the magnetospheric equator. The ratio between the wave amplitudes in conjugate points depend on the contrast between the ionospheric and magnetospheric conductances in these points. However, there are many factors that may obscure the observed ratio [Hartinger et al., 2017].

\section{Conclusion}

The mapping of the auroral particle precipitation, derived from the OVATION-prime model, and Pc5 spectral power has shown that the wave power in the morning sector is localized at the equatorward slope of the auroral monoenergetic electron precipitation, far poleward from the diffuse electron precipitation. This observational result confirms the effect earlier described: spatial/temporal variations in Pc5 wave power are closely related to the location and intensity of the auroral electrojet and magnetospheric fieldaligned currents. From the observed relationship between ULF wave power and the auroral boundaries it may be concluded that this region of the auroral oval is a preferred latitude for magnetospheric fieldline resonator excitation. An adequate interpretation may require a substantial augmentation of existing Pc5 models.

\section{Acknowledgements.}

This study is supported by the NSF grant AGS1264146 (MJE) and grant 246725 from the POLARPROG program of the Norwegian Research Council (VAP,DAL). The OVATION Prime model was developed at Johns Hopkins Applied Physics Laboratory by $P$. Newell and co-workers. The model IDL code is freely downloaded from the SourceForge website. Useful comments of the reviewer are appreciated. 


\section{References}

Chen, L. and A. Hasegawa, A theory of long period magnetic pulsations 1. Steady state excitation of field line resonances, J. Geophys. Res., 1024-1032, 1974.

Hartinger, M.D., D.L. Turner, F. Plaschke, V. Angelopoulos, and H.J. Singer, The role of transient ion foreshock phenomena in driving Pc5 ULF wave activity, J. Geophys. Res., doi:10.1029/2012JA018349, 2013.

Hartinger, M.D., Z. Xu, C.R. Clauer, Y. Yu, D.R. Weimer, H. Kim, V. Pilipenko, D.T. Welling, R. Behlke, and A.N. Willer, Associating ground magnetometer observations with current or voltage generators, J. Geophys. Res. Space Physics, 122, doi:10.1002/2017JA024140, 2017.

Kessel, R.L., I.R. Mann, S.F. Fung, D.K. Milling, and N. Connell, Correlation of Pc5 wave power inside and outside the magnetosphere during high speed streams, Ann. Geophys., 22, 629- 641, 2004.

Kleimenova, N.G., O.V. Kozyreva, M. Bitterly, and J.-J. Schott, Long-period (1-6 mHz) geomagnetic pulsations during growth phase of large magnetic storm Feb. 21, 1994, Geomagn. Aeronomy, 40, 16-25, 2000.

Kozyreva, O.V., V.A. Pilipenko, M.J. Engebretson, D.Yu. Klimushkin, and P.N. Mager, Correspondence between the ULF wave power distribution and auroral oval, SolarTerrestrial Physics, 2, №2, 46-65, 2016.

Lepidi, S., and P. Francia, Low-frequency (1-4 mHz) geomagnetic field fluctuation power at different latitudes for a diagnosis of the auroral oval position, Memorie Soc. Astron. Italiana, 74, 762-765, 2003.

Lyons, R.L., T. Nagai, G.T. Blanchard, J.C. Samson, T. Yamamoto, T. Mukai, A. Nishida, and S. Kokubun, Association between Geotail plasma flows and auroral poleward boundary intensifications observed by CANOPUS photometers, J. Geophys. Res., 104, 4485-4500, 1999.

Lam, H.L., and G. Rostoker, The relationship of Pc5 micropulsation activity in the morning sector to the auroral westward electrojet, Planetary Space Sci., 26, 473-492, 1978.

Mann, I.R., K.R. Murphy, L.G. Ozeke, I.J. Rae, D.K. Milling, A. Kale, and F. Honary, The role of ultralow frequency waves in radiation belt dynamics, in: Dynamics of the Earth's Radiation Belts and Inner Magnetosphere, Geophys. Monogr. Ser., vol. 199, ed. D. Summers, 69-91, AGU, Washington DC, 2012.

Newell, P.T., T. Sotirelis, and S. Wing, Diffuse, monoenergetic, and broadband aurora: The global precipitation budget, J. Geophys. Res., 114, A09207, doi:10.1029/2009JA014326, 2009.
Pilipenko, V., J. Watermann, V. Popov, and V. Papitashvili, Relationship between auroral electrojet and Pc5 ULF waves, J. Atmosph. Solar -Terr. Phys., 63, 1545-1557, 2001.

Pilipenko V.A., D.Yu. Klimushkin, P.N. Mager, M.J. Engebretson, and O.V. Kozyreva, Generation of resonant Alfven waves in the auroral oval, Annales Geophysicae, 34, 241-248, 2016.

Posch, J.L., M.J. Engebretson, V.A. Pilipenko, W.J. Hughes, C.T. Russell, and L.J. Lanzerotti, Characterizing the long period ULF response to magnetic storms, J. Geophys. Res., 108, 1029, doi:10.1029/2002JA009386, 2003.

Potemra, T.A., L.J. Zanetti, P.F. Bythrow, R.E. Erlandson, R. Lundin, G.T. Marklund, L.P. Block, and P.-A. Lindquist, Resonant geomagnetic field oscillations and Birkeland currents in the morning sector, J. Geophys. Res., 93, 26612674, 1988.

Rostoker, G., and H-L. Lam, A generation mechanism for Pc5 micropulsations in the morning sector, Planet. Space Sci., 26, 493-505, 1978.

Samson J.C., R. Rankin, and V.T. Tikhonchuk, Optical signatures of auroral arcs produced by field line resonances: comparison with satellite observations and modeling, Ann. Geophysicae, 21, 933-945, 2003.

Schott, J.-J., N.G. Kleimenova, J. Bitterly, and O.V. Kozyreva, The strong Pc5 geomagnetic pulsations in the initial phase of the great magnetic storm of March 24, 1991, Earth, Planets and Space, 50, 101-106, 1998.

Simms, L.E., M.J. Engebretson, J.L. Posch, and W.J. Hughes, Effects of the equatorward auroral boundary location and solar wind parameters on Pc5 activity at auroral zone stations: A multiple regression analysis, J. Geophys. Res., 111, A10217, doi:10.1029/2005JA011587, 2006.

Sutcliff, P.R., and G. Rostoker, Dependence of Pc5 micropulsation power on conductivity variations in the morning sector, Planet. Space Sci., 27, 631-642, 1979.

Walker, A.D.M., R.A. Greenwald, W.F. Stuart, and C.A. Green, STARE auroral radar observations of Pc5 geomagnetic pulsations, J. Geophys. Res. 84, 3373-3388, 1979.

Ziesolleck, C.W.S., and D.R. McDiarmid, Auroral latitude Pc5 field line resonances: quantized frequencies, spatial characteristics, diurnal variation, J. Geophys. Res., 99, 5817-5830, 1994. 